\title{
A REMARK ON POSITIVE TOPOLOGICAL ENTROPY OF $N$-BUFFER SWITCHED FLOW NETWORKS
}

XIAO-SONG YANG

Received 24 January 2005

We present a simpler elementary proof on positive topological entropy of the $N$-buffer switched flow networks based on a new simple theorem on positive topological entropy of continuous map on compact metric space.

\section{Introduction}

Recently, the $N$-buffer switched flow networks have received intensive investigations due to its significance in manufacturing systems and other engineering disciplines, as well as physical problems $[1,2,3,4,6,7,8,10]$. Various dynamical behaviours of this model, such as existence and stability of periodic trajectories, bifurcation and chaos were extensively investigated. In case of more than three buffers, [7] gave an elegant proof on existence of chaos in terms of positive entropy. However, to understand the arguments of [7], one has to know much knowledge about invariant SRB measure, the Markov partition and the relevant theory on entropy of ergodic Markov shift, this make it difficult to catch on for readers less of good background of ergodic theory in dynamical systems.

The author presented in [10] an elementary proof on the positivity of the entropy in the 3-buffer switched flow networks. Motivated by the work [10], the author obtained in [8] a proof of positive entropy of the $N$-buffer switched flow networkswhere the arguments are of geometric flavour and easy to understand even for readers who are not familiar with modern theory of dynamical systems.

However, the proof given in [8] is somewhat tedious. In this paper, we will present a simpler proof of positive topological entropy of the $N$-buffer switched flow networks. The proof is based on a new simple result on positive topological entropy of continuous map on compact metric space established in this paper.

\section{Symbolic dynamics and some preliminaries}

First we recall some aspects of dynamical systems and symbolic dynamics.

Let $S_{m}=\{1, \ldots, m\}$ be the set of nonnegative successive integer from 1 to $m$. Let $\Sigma_{m}$ be the collection of all one-sided sequences with their elements belonging to $S_{m}$, that is, 
every element $s$ of $\Sigma_{m}$ is of the following form

$$
s=\left\{s_{1}, \ldots, s_{n}, \ldots\right\}, \quad s_{i} \in S_{m} .
$$

Now consider another sequence $\bar{s} \in \Sigma_{m}$

$$
\bar{s}=\left\{\bar{s}_{1}, \ldots, \bar{s}_{n}, \ldots\right\}, \quad \bar{s}_{i} \in S_{m} .
$$

The distance between $s$ and $\bar{s}$ is defined as

$$
d(s, \bar{s})=\sum_{i=1}^{\infty} \frac{1}{2^{i}} \frac{\left|s_{i}-\bar{s}_{i}\right|}{1+\left|s_{i}-\bar{s}_{i}\right|} .
$$

With the distance defined above, $\Sigma_{m}$ is a metric space. In addition, $\Sigma_{m}$ is compact, totally disconnected and perfect. A set having these properties is often defined as a Cantor set, such a Cantor set frequently appears in characterization of complex structure of invariant set in a chaotic dynamical system. For more detailed discussions on $\Sigma_{m}$, see [5].

Furthermore, define the $m$-shift map $\sigma: \Sigma_{m} \rightarrow \Sigma_{m}$ as follows

$$
\sigma(s)_{i}=s_{i+1}
$$

Then there are the following facts.

(a) $\sigma\left(\Sigma_{m}\right)=\Sigma_{m}$ and $\sigma$ is continuous.

(b) The shift map $\sigma$ as a dynamical system defined on $\Sigma_{m}$ has the following properties:

(i) $\sigma$ has a countable infinity of periodic orbits consisting of orbits of all periods;

(ii) $\sigma$ has an uncountable infinity of aperiodic orbits; and

(iii) $\sigma$ has a dense orbit.

For proofs of the above statements, we refer the reader to [5]. A consequence of statement (b) is that the dynamics generated by the shift map $\sigma$ is sensitive to initial conditions therefore is chaotic.

Next we recall the semi-conjugacy in context of a continuous map and the shift map $\sigma$, which is conventionally defined as follows

Definition 2.1. Let $X$ be a metric space. Consider a continuous map $f: X \rightarrow X$. Let $\Lambda$ be a compact invariant set of $f$. If there exists a continuous surjective map

$$
h: \Lambda \longrightarrow \Sigma_{m}
$$

such that $h \circ f=\sigma \circ h$, then the restriction of $f$ to $\Lambda, f \mid \Lambda$ is said to be semi-conjugate to $\sigma$.

The following result is useful for the sequel arguments of the main result of this paper, we restate a version of it as a lemma for reader's convenience. 
Lemma 2.2. Let $X$ be a metric space, $D$ is a compact subset of $X$, and $f: D \rightarrow X$ is map satisfying assumption that there exist $m$ mutually disjoint compact subsets $D_{1}, \ldots$, and $D_{m}$ of $D$, the restriction of $f$ to each $D_{i}$, that is, $f \mid D_{i}$ is continuous. Suppose that

$$
f\left(D_{j}\right) \supset \bigcup_{i=1}^{m} D_{i}, \quad j=1,2, \ldots, m
$$

then there exists a compact invariant set $\Lambda \subset D$, such that $f \mid \Lambda$ is semi-conjugate to the m-shift map.

The proof is very easy, for a proof see [9].

Lemma 2.3 [5]. Let $X$ be a compact metric space, and $f: X \rightarrow X$ a continuous map. If there exists an invariant set $\Lambda \subset X$ such that $f \mid \Lambda$ is semi-conjugate to the $m$-shift $\sigma$, then

$$
h(f) \geq h(\sigma)=\log m
$$

where $h(f)$ denotes the entropy of the map $f$. In addition, for every positive integer $k$,

$$
h\left(f^{k}\right)=k h(f)
$$

In the following we recall the concept of topological entropy for reader's convenience. Definition 2.4. A set $E \subset X$ is called $(n, \varepsilon)$-separated if for every two different points $x, y \in$ $E$, there exists $0 \leq j<n$ such that the distance between $f^{j}(x)$ and $f^{j}(y)$ is greater than $\varepsilon$. Now let the number $s(n, \varepsilon)$ denotes the cardinality of a maximum $(n, \varepsilon)$-separated set:

$$
s(n, \varepsilon)=\max \{\operatorname{card} E: E \text { is }(n, \varepsilon) \text {-separated }\} .
$$

The topological entropy of the map $f$ is defined as

$$
h(f)=\lim _{\varepsilon \rightarrow 0} \limsup \frac{1}{n} \log s(n, \varepsilon) .
$$

For the notions and discussions on entropy of dynamical systems, the reader can refer [5]. We just recall a result stated in Lemma 2.3, which will be used in this paper.

\section{Proof of positive entropy of $N$-buffer switched flow networks}

To give a simpler proof of positive entropy of the $N$-buffer switched flow network, we first give the following result that is fundamental for our new proof.

Theorem 3.1. Let $X$ be a metric space, $D$ is a compact subset of $X, D_{i}, j=1,2, \ldots, m$, is a subset of $D$, and $f: D \rightarrow X$ is a continuous map satisfying the following assumptions:

(1) for each pair $i \neq j, 1 \leq i, j \leq m, f\left(D_{i} \cap D_{j}\right) \subseteq D_{i} \cap D_{j}$;

(2) the intersection $D_{1} \cap \cdots \cap D_{m}$ is empty;

(3) $f\left(D_{j}\right) \supset \bigcup_{i=1}^{m} D_{i}-D_{j}, j=1,2, \ldots, m$ 
then there exists a compact invariant set $\Lambda \subset D$, such that $f^{m-1} \mid \Lambda$ is semi-conjugate to $(m-1)$-shift dynamics. And

$$
h(f) \geq \frac{1}{m-1} \log (m-1)
$$

Proof. The proof is very easy. Without loss of generality let us consider the subset $D_{1}$ and study the dynamics of the restricted map $f \mid D_{1}$. We are going to find $m-1$ mutually disjointed subsets contained in $D_{1}$, such that Lemma 2.2 can be applied.

For this purpose let $\tilde{N}=\{2,3, \ldots, m\}$, the set of integers from 2 to $m$. Consider the following matrix with elements in $\tilde{N}$

$$
\left[\begin{array}{cccc}
2 & 3 & \cdots & m \\
3 & \cdots & m & 2 \\
\vdots & \vdots & \vdots & \vdots \\
m-1 & m & \cdots & m-2
\end{array}\right]
$$

This matrix is obtained as follows.

Let $J=i_{1} i_{2}, \ldots, i_{m-1}$ be a row in the above matrix, say, the $j$ th row, then the $(j+1)$ th row is obtained by the permutation map $P$ defined as:

$$
P(I)=i_{2}, \ldots, i_{m-2} i_{m-1} i_{1} .
$$

Now consider $m-1$ sequences obtained from each column of (3.2)

$$
I_{1}=23, \ldots, m-1, I_{2}=3, \ldots, m-1, m, \ldots, I_{m-1}=m 2, \ldots, m-2 .
$$

Consider the following compact subsets of $D_{1}$;

$$
D_{I_{k}}^{1}, \quad k=1,2, \ldots, m-1 .
$$

They are constructed as follows:

Note that in each sequence $I_{k}=k_{1} k_{2}, \ldots, k_{m-2}, k=1,2, \ldots, m-1$, there are no two numbers that are the same and 1 is not in every such sequence. Clearly, $f\left(D_{k_{m-2}}\right) \supset D_{1}$ in view of assumption (3). Then it is easy to see that there exists a compact subset $\bar{D}_{k_{m-2}} \subset D_{k_{m-2}}$ such that $f\left(\bar{D}_{k_{m-2}}\right)=D_{1}$. Since $k_{m-3} \neq k_{m-2}, f\left(D_{k_{m-3}}\right) \supset D_{k_{m-2}}$, this implies that there exists a compact subset $\bar{D}_{k_{m-3} k_{m-2}} \subset D_{k_{m-3}}$ such that $f\left(\bar{D}_{k_{m-3} k_{m-2}}\right)=D_{k_{m-2}}$. Continuing this way, we get a sequence of subsets $\bar{D}_{k_{m-h-2}, \ldots, k_{m-2}} \subset D_{k_{m-h-2}}$ such that $f\left(\bar{D}_{k_{m-h-2}, \ldots, k_{m-2}}\right)=$ $\bar{D}_{k_{m-h-1}, \ldots, k_{m-2}}, h=1, \ldots, m-3$. Finally, consider the compact subset $D_{I_{k}}^{1} \subset D_{1}$ such that

$$
f\left(D_{I_{k}}^{1}\right)=\bar{D}_{I_{k}}=\bar{D}_{k_{1}, \ldots, k_{m-2}}, \quad k=1,2, \ldots, m-1
$$

Then it is easy to see that $f^{m-1}\left(D_{I_{k}}^{1}\right)=D_{1}$.

Now it remains to show that the intersection of $D_{I_{l}}^{1}$ with $D_{I_{k}}^{1}$ is empty if $I_{k} \neq I_{l}$. To this end, suppose that this is not the case, then consider a point $x \in D_{I_{k}}^{1} \cap D_{I_{l}}^{1}$, then

$$
f(x) \in \bar{D}_{I_{k}} \cap \bar{D}_{l_{k}} \subset D_{k_{1}} \cap D_{l_{1}} \text {. }
$$


Now in view of assumption (1) and the way of constructing sequences (3.4), it follows that

$$
f^{m-1}(x) \in D_{1} \cap \cdots \cap D_{m}
$$

which is in contradiction to assumption (2). Finally, in view of Lemmas 2.2 and 2.3, we have the inequality (3.1).

Now we present a simpler proof of positive entropy of the $N$-buffer switched flow network. Consider a system of $N$ buffers and one server. In such a system, work is removed from buffer $i$ at a fixed rate of $\rho_{i}>0$ while the server delivers material to a selected buffer at a unit rate. The control law is applied to the server so that once a buffer empties, the server instantaneously starts to fill the empty buffer. The system is assume to be close in the sense that

$$
\sum_{i=1}^{N} \rho_{i}=1
$$

Let $x_{i}(t)$ be the amount of work in buffer $i$ at time $t \geq 0$, and $x(t)=\left(x_{1}(t), \ldots, x_{N}(t)\right)$ denote the state of work of the buffers at time $t$, then

$$
\sum_{i=1}^{N} x_{i}(t)=1
$$

if it is assumed that $\sum_{i=1}^{N} x_{i}(0)=1$.

Consider the sample sequence at clearing time, $\left\{t_{n}\right\}$, which are the times when at least one of these buffers empty. Let $x(n)=\left(x_{1}\left(t_{n}\right), \ldots, x_{N}\left(t_{n}\right)\right)$, then the sequence $\{x(n)\}$ evolves on the $N-2$ dimensional manifold

$$
X=\left\{x: \sum_{i=1}^{N} x_{i}=1, x_{i} \geq 0, \exists 1 \leq j \leq N, x_{j}=0\right\}
$$

by the following rule $G: X \rightarrow X$ :

$$
x(n+1)=G(x(n))
$$

$G(x)$ is defined as follows:

(1) $G(x)=x$, if at least two of the buffers empty at the same time,

(2) $x(n+1)=G(x(n))=x(n)+\min _{i \neq j}\left(x_{i}(n) / \rho_{i}\right)\left(1_{j}-\rho\right)$, otherwise,

where $1_{j}$ is a vector with all zeros except for one in the $j$ th position and $\rho$ is a vector of work rates $\rho_{j}$. It is apparent that $X$ defined above can be regarded as the surface of the standard $(N-1)$ simplex $\phi$ defined by

$$
\phi=\left\{x \in R^{N}: x=\sum_{i=1}^{N} x_{i} e_{i}, x_{i} \geq 0, \sum_{i=1}^{N} x_{i}=1\right\},
$$

where $e_{1}=(1,0, \ldots, 0), e_{2}=(0,1,0, \ldots, 0), \ldots, e_{N}=(0,0, \ldots, 1)$, and they are the vertices of the piecewise linear manifold $X$. 
Let $X_{i} \subset X$ be the $i$ th face of $X$ :

$$
X_{i}=\left\{x \in X: x_{i}=0\right\}, \quad i=1,2, \ldots, N .
$$

It is very easy to see that the map $G$ has the following properties.

The restriction of the map $G$ to every face $X_{j}$, that is,

$$
G \mid X_{j}: X_{j} \longrightarrow X-\hat{X}_{j}
$$

is a continuous surjective (i.e. onto)map. Here $\hat{X}_{j}=X_{j}-\partial X_{j}\left(\partial X_{j}\right.$ is the boundary of $\left.X_{j}\right)$, that is, the set consists of interior points of $X_{j} . G\left(X_{i} \cap X_{j}\right)=X_{i} \cap X_{j}, i \neq j, 1 \leq i, j \leq N$.

In view of the above theorem (Theorem 3.1) it is easy to prove the following result.

Corollary 3.2. The map $G: X \rightarrow X$ is chaotic and its entropy, $h$, satisfies the following inequality

$$
h(G) \geq \frac{1}{N-1} \log (N-1) .
$$

Thus (3.12) is chaotic.

\section{Conclusion}

In this paper, we have discussed the topological entropy of the dynamical system described by continuous maps defined on a compact metric space, and obtained a simple new result. Based on this result we have presented very simple proof of positive topological entropy of the so-called $N$-buffer switched flow networks.

\section{Acknowledgment}

This work is partially supported by Talents Foundation of Huazhong University of Science and Technology (0101011092) and the Program for New Century Excellent Talents in University.

\section{References}

[1] A. Amann, K. Peters, U. Parlitz, A. Wacker, and E. Schöll, Hybrid model for chaotic front dynamics: from semiconductors to water tanks, Phys. Rev. Lett. 91 (2003), no. 6, 066601.

[2] C. Chase, J. Serrano, and P. J. Ramadge, Periodicity and chaos from switched flow systems: contrasting examples of discretely controlled continuous systems, IEEE Trans. Automat. Control 38 (1993), no. 1, 70-83.

[3] C. Horn and P. J. Ramadge, A topological analysis of a family of dynamical systems with nonstandard chaotic and periodic behaviour, Internat. J. Control 67 (1997), no. 6, 979-996.

[4] K. Peters and U. Parlitz, Hybrid systems forming strange billiards, Internat. J. Bifur. Chaos Appl. Sci. Engrg. 13 (2003), no. 9, 2575-2588.

[5] C. Robinson, Dynamical Systems: Stability, Symbolic Dynamics, and Chaos, Studies in Advanced Mathematics, CRC Press, Florida, 1995.

[6] A. V. Savkin and A. S. Matveev, A switched server system of order $n$ with all its trajectories converging to ( $n-1)$ ! limit cycles, Automatica J. IFAC 37 (2001), no. 2, 303-306. 
[7] T. Schürmann and I. Hoffmann, The entropy of "strange" billiards inside n-simplexes, J. Phys. A 28 (1995), no. 17, 5033-5039.

[8] X.-S. Yang, An estimate of topological entropy of N-buffer fluid networks, Nonlinearity 18 (2005), no. $1,263-275$.

[9] X.-S. Yang and Y. Tang, Horseshoes in piecewise continuous maps, Chaos Solitons Fractals 19 (2004), no. 4, 841-845.

[10] A note on entropy of 3-buffer flow model, Chaos Solitons Fractals 23 (2005), no. 1, 87-91.

Xiao-Song Yang: Department of Mathematics, Huazhong University of Science and Technology, Wuhan 430074, China; Department of Control Science and Engineering, Huazhong University of Science and Technology, Wuhan 430079, China

E-mail address: yangxs@cqupt.edu.cn 


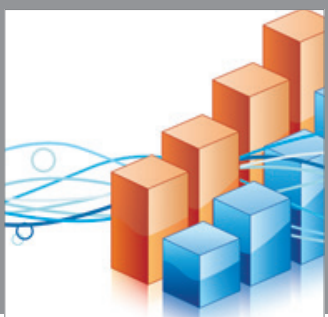

Advances in

Operations Research

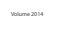

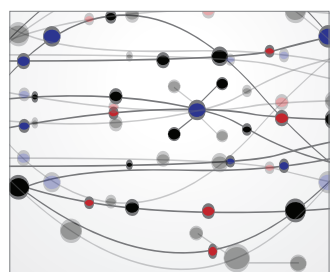

\section{The Scientific} World Journal
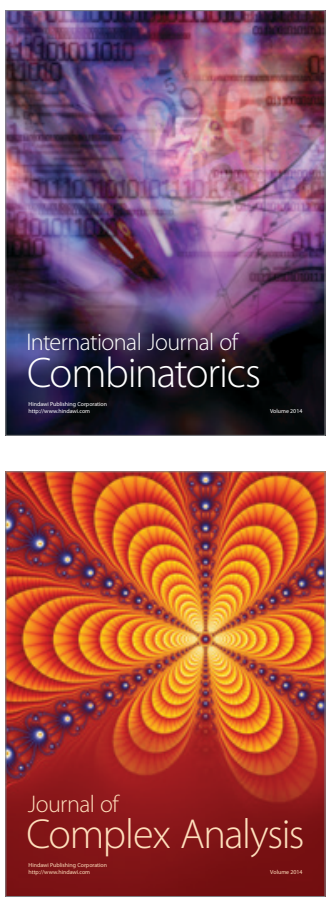

International Journal of

Mathematics and

Mathematical

Sciences
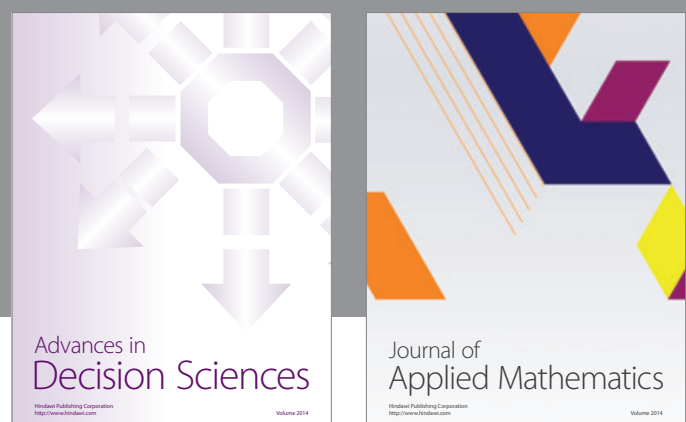

Journal of

Applied Mathematics
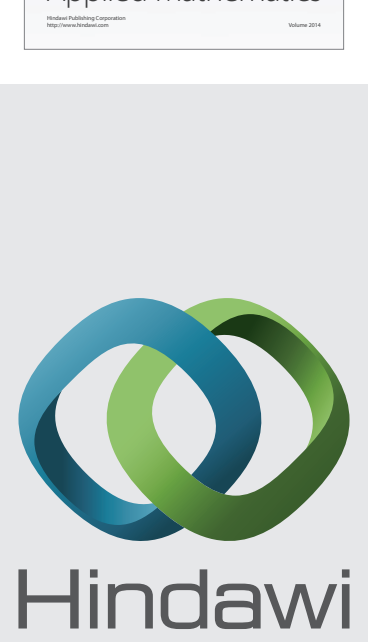

Submit your manuscripts at http://www.hindawi.com
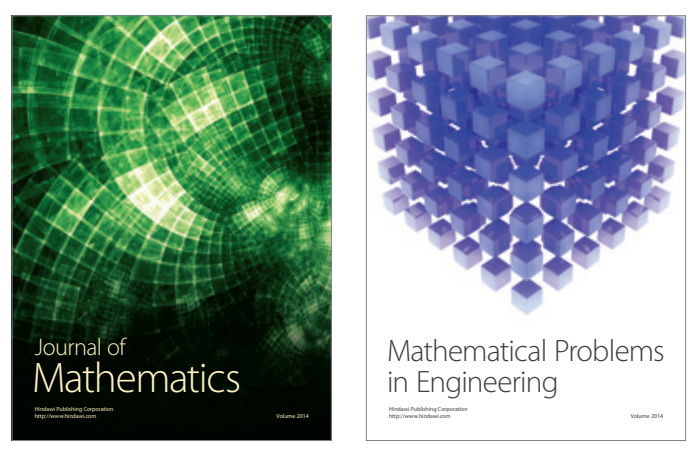

Mathematical Problems in Engineering
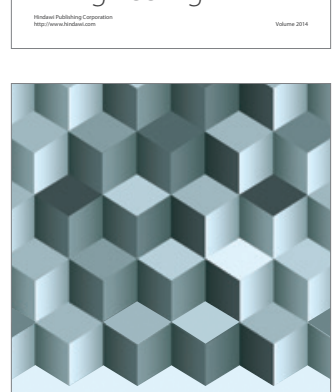

Journal of

Function Spaces
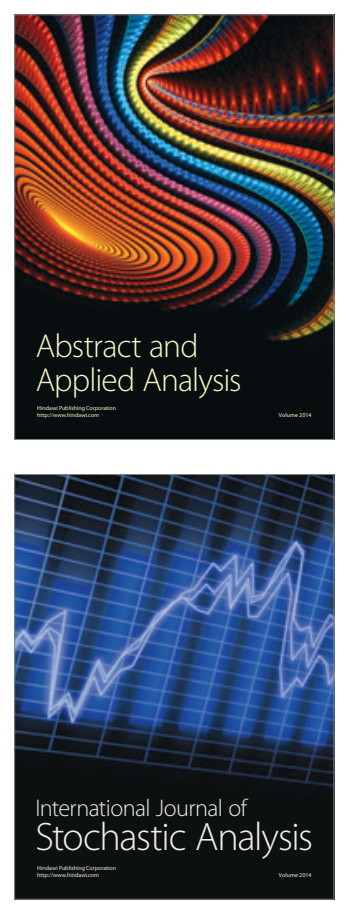

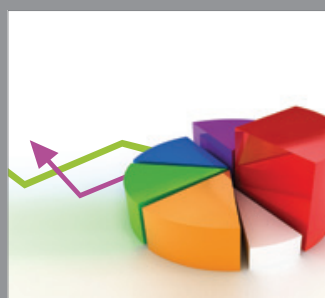

ournal of

Probability and Statistics

Promensencen
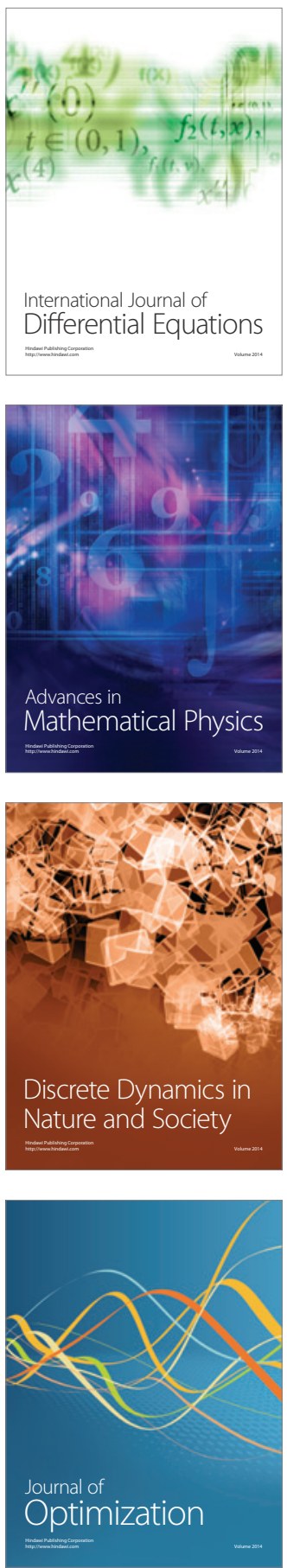